\title{
实验增温对藏北高寒草甸植物繁殖物候的影响
}

朱军涛

中国科学院地理科学与资源研究所, 生态系统网络观测与模拟重点实验室, 拉萨高原生态试验站, 北京 100101

\begin{abstract}
摘 要 全球气候变暖对高寒和极地地区的植物物候产生强烈的影响。该研究主要关注增温条件下藏北高寒草甸不同功能型 植物繁殖时间(生殖物候)的改变。实验采用开顶箱式增温方法, 对3个主要功能群浅根-早花、浅根-中花和深根-晚花植物的现 蕾、开花、结实时间进行观测。研究结果表明: (1)增温导致了土壤水分胁迫, 显著推迟了浅根-早花植物高山嵩草(Kobresia pygmaea)的繁殖时间; (2)增温显著提前了浅根-中花植物钉柱委陵菜(Potentilla saundersiana)和深根晚花植物紫花针茅(Stipa purpurea)和矮羊茅(Festuca coelestis)的繁殖时间; (3)增温没有显著影响浅根-中花植物楔叶委陵菜(Potentilla cuneata)和深根晚花植物无茎黄鹤菜(Youngia simulatrix)的繁殖时间; (4)增温缩短了3种类型植物的开花持续时间。这些结果显示增温改变了 藏北高寒草甸群落中多数物种的繁殖时间, 这预示着在未来更热更干的生长季, 青藏高原高寒草甸系统的植物物候格局可能 会被重塑。
\end{abstract}

关键词 高寒草甸; 实验增温; 物候偏移; 繁殖物候; 青藏高原

引用格式: 朱军涛 (2016). 实验增温对藏北高寒草甸植物繁殖物候的影响. 植物生态学报, 40, 1028-1036. doi: 10.17521/cjpe.2016.0068

\section{Effects of experimental warming on plant reproductive phenology in Xizang alpine meadow}

ZHU Jun-Tao*

Lhasa Plateau Ecosystem Research Station, Key Laboratory of Ecosystem Network Observation and Modeling, Institute of Geographic Sciences and Natural Resources Research, Chinese Academy of Sciences, Beijing 100101, China

\section{Abstract}

Aims Climate warming strongly influences reproductive phenology of plants in alpine and arctic ecosystems. Here we focus on phenological shifts caused by warming in a typical alpine meadow on the Qinghai-Xizang Plateau. Our objective was to explore phenological responses of alpine plant species to experimental warming.

Methods Passive warming was achieved using open-top chambers (OTCs). The treatments included control (C), and four levels of warming (T1, T2, T3, T4). We selected Kobresia pygmaea, Potentilla saundersiana, Potentilla cuneata, Stipa purpurea, Festuca coelestis and Youngia simulatrix as the focal species. Plant phenology was scored every 3-5 days in the growing season. The reproductive phenology phases of each species were estimated through fitting the phenological scores to the Richards function.

Important findings Under soil water stress caused by warming, most plants in the alpine meadow advanced or delayed their reproductive events. As a result, warming significantly delayed phenological development of $K$. pygmaea. Warming significantly advanced reproductive phenology of P. saundersiana, S. purpurea and F. coelestis, but not of $P$. cuneata and $Y$. simulatrix. In addition, warming significantly shortened the average flowering duration of alpine plant species. The potentially warmer and drier growing seasons under climate change may shift the reproductive phenology of the alpine systems in similar pattern.

Key words alpine meadow; experimental warming; phenological shifts; reproductive phenology; Qinghai-Xizang Plateau

Citation: Zhu JT (2016). Effects of experimental warming on plant reproductive phenology in Xizang alpine meadow. Chinese Journal of Plant Ecology, 40, 1028-1036. doi: 10.17521/cjpe.2016.0068

联合国政府间气候变化专门委员会(IPCC)第4 次评估报告指出，未来50年内全球气候变化将长期
影响草原的物种组成、群落演替和生物多样性, 使 其偏离原有状态(IPCC, 2007)。这其中植物物候将首

收稿日期Received: 2016-02-22 接受日期Accepted: 2016-05-09

*E-mail: zhujt@igsnrr.ac.cn 
先响应气候变化, 偏离其原有状态, 改变植物群落 原有的物候格局(Sherry et al., 2007), 导致群落种间 关系重新组合(Springate \& Kover, 2014), 进而对生 态系统的结构和功能产生深远影响(Visser \& Both, 2005)。植物物候作为气候变暖的“指纹”, 对温度升 高的响应尤为敏感, 且不同功能群植物(早花和晚 花植物)的物候及物候的各个阶段(现蕾、开花和结 实等)对气候变暖的响应不同。

在高寒地区, 温度升高可以缓减低温对植物生 长发育带来的限制作用, 促进植物生长(阿舍小虎, 2013), 但是与此同时, 土壤水分蒸发和植物蒸腾作 用的提高对植物生长发育带来不利影响(Dorji et al., 2013)。因此, 在极地和高寒地区, 温度升高和土壤 水分减少两者的共同作用导致得出不同的研究结 论, 增温使繁殖物候提前(Cleland et al., 2006)、推迟 (Yu et al., 2010)或没有影响(Hoffmann et al., 2010)。 而最近的研究更是提出实验增温可能过低地估计了 植物物候对气候变化的响应, 原因是增温的同时引 起了土壤干旱(Rutishauser et al., 2012)。

植物的形态特征、生理特征及生活史性状等调 节着植物物候对增温的响应 (Hoffmann et al., 2010)。例如在干旱和半干旱地区, 由于灌木的根系 发达, 能利用深层的土壤水分, 因此增温可能使灌 木的花期物候提前(Dunne et al., 2003); 而增温导致 的土壤水分减少, 将制约浅根植物的生长发育 (Klein et al., 2008)。在北美高草草原, 增温使早花物 种开花提前而晚花物种开花延迟 (Sherry et al., 2007)。总之, 植物的性状和生活史特征, 比如根系 深度、开花早晚等, 在植物繁殖物候响应气候变化 过程中有至关重要的作用(Dorji et al., 2013; Wang et al., 2014)。

不同种类植物物候对增温的响应快慢不同 (Hoffmann et al., 2010), 一些种类的物候会快速响 应增温, 而另一些种类可能需要增温的累积效应 (Arft et al., 1999; Hoffmann et al., 2010)。例如一些研 究指出早花植物可能比晚花植物对增温的响应更敏 感(Wolkovich et al., 2012), 但也有一些相反的结论, 例如在北美高草草原中夏季开花的植物对增温的响 应更敏感(Sherry et al., 2007)。另外, 植物物候的各 个阶段对环境变化的响应并不同步, 不同的物候期 对增温的响应不同(Post et al., 2008)。例如与其他物 候期相比, 现蕾和开花期对增温的响应更敏感
(Hoffmann et al., 2010)。

综上所述, 前人已开展了大量有关植物物候对 全球气候变暖的响应的研究, 但在青藏高原此类研 究还相对较少。因此, 本研究利用气候变暖模拟控 制实验, 研究温度升高对藏北高寒草甸植物繁殖物 候的影响。在植物的生长季 5-9月, 采用开顶箱 (OTCs)式增温的方法对藏北高寒草甸进行增温, 实 验分为对照、增温( T1、T2、T3、T4) 5种处理, 并 将植物分为 3 个主要功能群: 浅根-早花、浅根-中花 和深根-晚花植物。通过观测和记录不同功能群植物 的现蕾、开花、结实的时间，同时测定空气和土壤 温湿度等, 旨在探寻并揭示不同功能群植物繁殖时 间对增温的响应规律。本研究将为高寒草甸生态系 统响应未来气候变暖提供基础数据和理论依据, 对 于未来气候变暖下青藏高原的生态环境变化预测、 生态安全建设及牧业生产等具有重大的科学价值。

\section{1 材料和方法}

\section{1 研究区概况}

本研究地点位于藏北高原草地生态系统研究站 (简称那曲站)。那曲站 $\left(31.64^{\circ} \mathrm{N}, 92.02^{\circ} \mathrm{E}\right.$, 海拔 $4585 \mathrm{~m}$ )隶属于中国科学院地理科学与资源研究所 拉萨高原生态实验站, 位于藏北㒸塘高原核心地带 那曲地区那曲县那曲镇曲果仁村境内, 距那曲县城 约 $22 \mathrm{~km}$ 。该地区属于高原亚寒带季风半湿润气候 区。平均海拔 $4500 \mathrm{~m}$ 以上, 高寒缺氧, 气候干燥。 全年大风日 100 天左右, 平均风速 $2.7 \mathrm{~m} \cdot \mathrm{s}^{-1}$, 最大风 速可达 $26.3 \mathrm{~m} \cdot \mathrm{s}^{-1}$; 年平均气温 $-1.2{ }^{\circ} \mathrm{C}$, 全年日照时 间2 $788 \mathrm{~h}$; 年降水量430 mm, 集中在6-9月, 占全 年降水量的 $85 \%$ 。过去 60 年间夏季季风平均开始的 时间为5月22日(day of year, DOY: 142), 且具有较强 的年际波动。全年没有绝对无霜期, 每年10月至次 年5月为风雪期和土壤冻结期, 6月至 9 月为植物生长 期。

植被类型为典型的高寒草甸, 优势种为高山嵩 草(Kobresia pygmaea), 常见伴生种有钉柱委陵菜 (Potentilla saundersiana)、楔叶委陵菜(Potentilla cuneata)、二裂委陵菜(Potentilla bifurca)、紫花针茅 (Stipa purpurea)、矮羊茅(Festuca coelestis)、无茎黄 鹤菜(Youngia simulatrix)、高山风毛菊(Saussurea alpina)、早熟禾(Poa annua)、矮火线草(Leontopodium nanum)等, 植被覆盖度60\%-90\%。土壤类型 
为高寒草甸土。

\section{2 实验设计}

本实验研究对象为自然状态下的高寒草甸生态 系统, 采用OTCs式增温装置。利用OTCs的高度控 制增温的效果，设置了4个增温处理(T1、T2、T3、 $\mathrm{T} 4$ ), 与对照相比, 2015年生长季 T1、T2、T3、T4分 别使空气温度提高了 $2.2 、 2.8 、 3.2$ 和 $3.6{ }^{\circ} \mathrm{C}$ 。每个 OTCs的顶边长均为 $80 \mathrm{~cm}$, 保证所有处理的OTCs开 口大小一致, 底边长分别为 $100 、 110 、 120 、 130 \mathrm{~cm}$, 高度分别为 $40 、 60 、 80 、 100 \mathrm{~cm}$ 。OTCs均采用三角 钢支撑, 四周材质为透明玻璃纤维, 透光率达到 $90 \%$ 以上。因此, 本实验设置5种处理, 包括对照、 增温( $\mathrm{T} 1 、 \mathrm{~T} 2 、 \mathrm{~T} 3 、 \mathrm{~T} 4)$, 每个处理设置 4 个重复, 共 计 20 个样方。于 2013 年9月在那曲站完成OTCs安装。 利用HMP155A 探头(Vaisala, Helsinki, Finland)记录 地上 $10 \mathrm{~cm}$ 的空气温湿度。利用CS655探头(Campbell Scientific, Logan, USA)记录地下 $5 \mathrm{~cm}$ 的土壤温湿 度。每个实验处理安装两套空气和土壤温湿度探头, 小气候数据为两套探头的平均值。

\section{3 物候观测}

本实验选择的物种有高山嵩草、钉柱委陵菜、 楔叶委陵菜、紫花针茅、矮羊茅、无茎黄鹤菜等6 种植物, 分为 3 个不同的植物功能群, 浅根-早花植 物(高山嵩草)、浅根-中花植物(钉柱委陵菜、楔叶委 陵菜)、深根-晚花植物(紫花针茅、矮羊茅、无茎黄 鹤菜)。在本系统中, 高山嵩草、钉柱委陵菜、楔叶 委陵菜的根系集中在 $0-10 \mathrm{~cm}$ 的土壤中, 紫花针茅、 矮羊茅、无茎黄鹤菜的根系深度可以达到 $20 \mathrm{~cm}$ 以 上。高山嵩草平均花期是6月1日(DOY: $155 \pm 0.8$ ), 钉柱委陵菜花期是6月18日(DOY: $168 \pm 0.4)$, 楔叶 委陵菜花期是6月22日(DOY: $172 \pm 1.1$ ), 紫花针茅 花期是7月12日(DOY: $193 \pm 0.5) 、$ 矮羊茅花期是7月 16日(DOY: $197 \pm 1.2$ )、无茎黄鹤菜花期是7月27日 (DOY: $208 \pm 0.4$ )。选定的6个物种是该群落的优势 物种和主要常见种, 盖度和生物量占群落总盖度和 生物量的 $85 \%$ 以上。

本实验采用物候评分方法对植物繁殖物候进行 观测和记录。在每个处理样方内选取高山嵩草15株, 其他物种6株, 按顺序标记。物候观测从2014年5月 下旬开始，到2014年9月中旬结束。观测频次为3-5 天1次。对于杂类草和禾草而言, 分别采用6分制 (Price \& Waser, 1998)和4分制(Dunne et al., 2003)对
物候状态进行打分。对于杂类草植物，植物繁殖物 候期可分为 6 个阶段: 0 , 未开花; 1 , 花芽出现; 2 , 开 花; 3, 花衰老; 4, 果实开始发育; 5, 果实开始散布; 6 , 果实全部脱落。对于禾草类, 植物繁殖物候期可 分为 5 个阶段: 0 , 小穗尚在苞叶内; 1 , 小穗已伸出 于苞叶外; 2 , 花粉囊或花柱已伸出; 3 , 种子正在发 育; 4 , 种子脱落。当植物物候记录到达6或 4 时, 终 止该物种的生殖物候观测。在物候观测时, 将每个 植株上花(或花序)的所有生殖物候状态分值进行非 加权平均, 记录为该植株个体的生殖物候分值 (Dunne et al., 2003; Sherry et al., 2007), 如同一植株 上同时出现不同的几个物候状态，应将各物候状态 的分值进行平均处理, 其平均值计为该植株的物候 分值(李元恒等, 2014)。

\section{4 物候计算}

3-5天的观测间隔难以准确获取物种的开花和 结果时间(Xia \& Wan, 2013), 因此, 通常采用统计 模型对物候分值进行模拟，例如线性回归模型 (Price \& Waser, 1998)、贝叶斯统计模型(Bjorkman et al., 2015)、Richards生长方程等(Richards, 1959)。 Richards生长方程已成功地描述了北美高草草原植 物物候对增温和增加降水的响应(Sherry et al., 2007)和我国温带草原植物物候对增温的响应(Xia \& Wan, 2013)。其方程为:

$$
Y=\frac{K}{\left(1+a \mathrm{e}^{-b X}\right)^{m}}
$$

式中, $K$ 为最大化的生长, 即物候打分时的最大值, 非禾本科为 6 分、禾本科为 4 分时的植物生长; $a$ 为被 评价物种开始观察的日期的启动参数; $b$ 为所观察 时期内的生殖物候速率; $m$ 为曲线形状变异参数。在 每一个生长季中, 每个物候的打分序列必须符合 Richards方程 $\left(R^{2}>0.97, p<0.05\right)$, 并且找到符合该 方程的最适合的估测参数, 确定每个样方不同物种 的 4 个参数, 就可以准确计算开花和结实时间, 4 个 参数的计算在MATLAB软件中实现, 主要采用缩张 算法, 运用矩阵进行非线性拟合(Gu et al., 1998)。

每个物候期对应日期的计算可以从上面的公式 转换求得, 转换公式如下:

$$
X=-\frac{1}{b} \ln \left(\frac{\sqrt[m]{\frac{K}{Y}}-1}{a}\right)
$$


依据上式计算每一物种不同处理下现蕾时间 (budding time)、开花时间(flowering time)、结实时 间 (fruiting time)和开花持续时间 (flowering duration)。其中: 现蕾时间规定为非禾本科和禾本科植 物的物候分值为 1 分时对应的日期; 开花时间规定 为非禾本科和禾本科植物物候分值为 2 分时对应的 日期; 结实时间规定为非禾本科、禾本科植物的物 候分值分别为 3 和 2.5 分时对应的日期; 非禾本科植 物的开花持续时间为从开花(2分时)到花衰老(3分 时)的持续时间, 禾本科的开花持续时间为从开花 $(2$ 分时)开始到结实(2.5分时)的持续时间。统计分析采 用双因素方差分析和Turkey检验, 分别检验增温处 理、植物种类及其交互作用对现蕾、开花和结果时 间及开花持续时间的影响, 差异显著性水平设定为 0.05。采用单因素方差分析, 检验增温处理对单个物 种物候期的影响, 差异显著性水平设定为 0.05 。以上 分析在SPSS version 19.0中进行, 植物物候的拟合 在Matlab 7.0 软件中进行。

\section{2 结果}

\section{1 实验增温对小气候的影响}

在藏北高原OTCs增温方式提高了空气和土壤 温度, 降低了土壤水分(图1)。与对照相比, 实验增 温 $\mathrm{T} 1 、 \mathrm{~T} 2 、 \mathrm{~T} 3$ 和 $\mathrm{T} 4$ 分别使空气温度提高了 2.2、2.8、 3.2、3.6 ${ }^{\circ} \mathrm{C}$, 分别提高了土壤温度 $0.9 、 1.9 、 2.1$ 和 2.1 ${ }^{\circ} \mathrm{C}$; 增温 $\mathrm{T} 1 、 \mathrm{~T} 2 、 \mathrm{~T} 3$ 和 $\mathrm{T} 4$ 分别使土壤湿度降低了 $3.1 \% 、 4.4 \% 、 7.2 \%$ 和 $8.7 \%$ 。增温对土壤营养并没有 产生显著影响(表1)。2014年研究区夏季季风开始的 时间为5月25日(DOY: 145), 略晚于多年平均开始 期(DOY: 142)。生长季降水量为460 mm, 高于多年 平均降水量 $(408 \mathrm{~mm})$, 最大降水量出现在7月6日 (DOY: 187) (图1)。

\section{2 实验增温对植物现蕾时间的影响}

双因素方差分析表明, 增温处理、植物种类及
其交互作用显著影响了植物的现蕾时间(表2, $p<$ 0.05)。单因素方差分析结果表明, 实验增温显著推 迟了高山嵩草的现蕾时间(图2), 在 T1、T2、T3、T4 处理下, 分别推迟了 $6.5 \pm 0.8 、 7.2 \pm 1.0 、 12.5 \pm 2.3$ 、 $17.4 \pm 2.5 \mathrm{~d}(p<0.05)$ 。与之相反, 实验增温使钉柱 委陵菜的现蕾时间显著提前(图2), 在 $\mathrm{T} 1 、 \mathrm{~T} 2 、 \mathrm{~T} 3 、$ $\mathrm{T} 4$ 处理下, 分别提前了 $3.5 \pm 0.5 、 4.1 \pm 0.8 、 7.8 \pm 1.1$ 、 $8.4 \pm 1.4 \mathrm{~d}(p<0.05)$ 。除了增温处理 $\mathrm{T} 1$, 增温处理 T2、T3、T4使紫花针茅和矮羊茅的现蕾时间显著提 前, 分别提前了 $3.4 \pm 0.3 、 3.5 \pm 0.4 、 7.2 \pm 1.0 \mathrm{~d}$ 和 3.1 $\pm 0.2 、 3.3 \pm 0.5 、 6.7 \pm 0.6 \mathrm{~d}(p<0.05)$ 。实验增温对 楔叶委陵菜和无茎黄鹤菜的现蕾时间没有显著影响 (图2, $p>0.05$ )。

\section{3 实验增温对植物开花时间的影响}

双因素方差分析表明, 增温处理对植物的开花 时间没有显著影响(表2, $p>0.05$ ), 而植物种类、增 温和物种的交互作用显著改变了植物的开花时间 (表2, $p<0.05$ )。与对照相比, 实验增温显著推迟了 高山嵩草的开花时间(图2), 在 T1、T2、T3、T4处理 下, 分别推迟了 $6.0 \pm 0.6 、 7.0 \pm 0.8 、 13.6 \pm 1.7 、 18.5$ $\pm 2.2 \mathrm{~d}(p<0.05)$ 。与之相反, 实验增温使钉柱委陵 菜的开花时间显著提前(图2), 在 $\mathrm{T} 1 、 \mathrm{~T} 2 、 \mathrm{~T} 3 、 \mathrm{~T} 4$ 处理下, 分别提前了 $3.8 \pm 0.8 、 4.0 \pm 0.8 、 8.2 \pm 1.0$ 、 $8.7 \pm 1.5 \mathrm{~d}(p<0.05)$ 。增温处理 T2、T3、T4使紫花 针茅和矮羊茅的现蕾时间显著提前, 分别提前了 3.6 $\pm 0.5 、 4.1 \pm 0.5 、 7.5 \pm 0.5 \mathrm{~d}$ 和 $3.5 \pm 0.4 、 4.0 \pm 0.3$ 、 $7.1 \pm 0.3 \mathrm{~d}(p<0.05)$ 。实验增温对楔叶委陵菜和无 茎黄鹤菜的开花时间没有产生显著影响(图2, $p>$ 0.05)。

\section{4 实验增温对植物结果时间的影响}

双因素方差分析表明, 增温处理对植物的结果 时间没有显著影响(表2, $p>0.05$ ), 而植物种类、增 温和物种的交互作用显著改变了植物的结果时间 (表2, $p<0.05$ )。与对照相比, 实验增温显著推迟了

表1 不同增温处理下土壤C、N、P 含量及C:N的差异(平均值沶准误差)

Table 1 Soil C, N, P contents and C:N for each treatments (mean $\pm S E$ )

\begin{tabular}{|c|c|c|c|c|}
\hline 处理 Treatment & 氮含量 N content (\%) & 碳含量 C content (\%) & 磷含量 P content $\left(\mathrm{mg} \cdot \mathrm{g}^{-1}\right)$ & $\mathrm{C}: \mathrm{N}$ \\
\hline 增温T4 Warming T4 & $0.22 \pm 0.04$ & $2.42 \pm 0.52$ & $0.75 \pm 0.04$ & $11.00 \pm 0.32$ \\
\hline 增温T3 Warming T3 & $0.24 \pm 0.06$ & $2.31 \pm 0.65$ & $0.73 \pm 0.05$ & $9.63 \pm 0.55$ \\
\hline 增温T2 Warming T2 & $0.21 \pm 0.05$ & $2.35 \pm 0.74$ & $0.74 \pm 0.04$ & $11.19 \pm 0.74$ \\
\hline 增温T1 Warming T1 & $0.25 \pm 0.07$ & $2.40 \pm 0.55$ & $0.72 \pm 0.03$ & $9.60 \pm 0.65$ \\
\hline 对照 Control & $0.25 \pm 0.06$ & $2.53 \pm 0.61$ & $0.75 \pm 0.04$ & $10.12 \pm 0.44$ \\
\hline
\end{tabular}



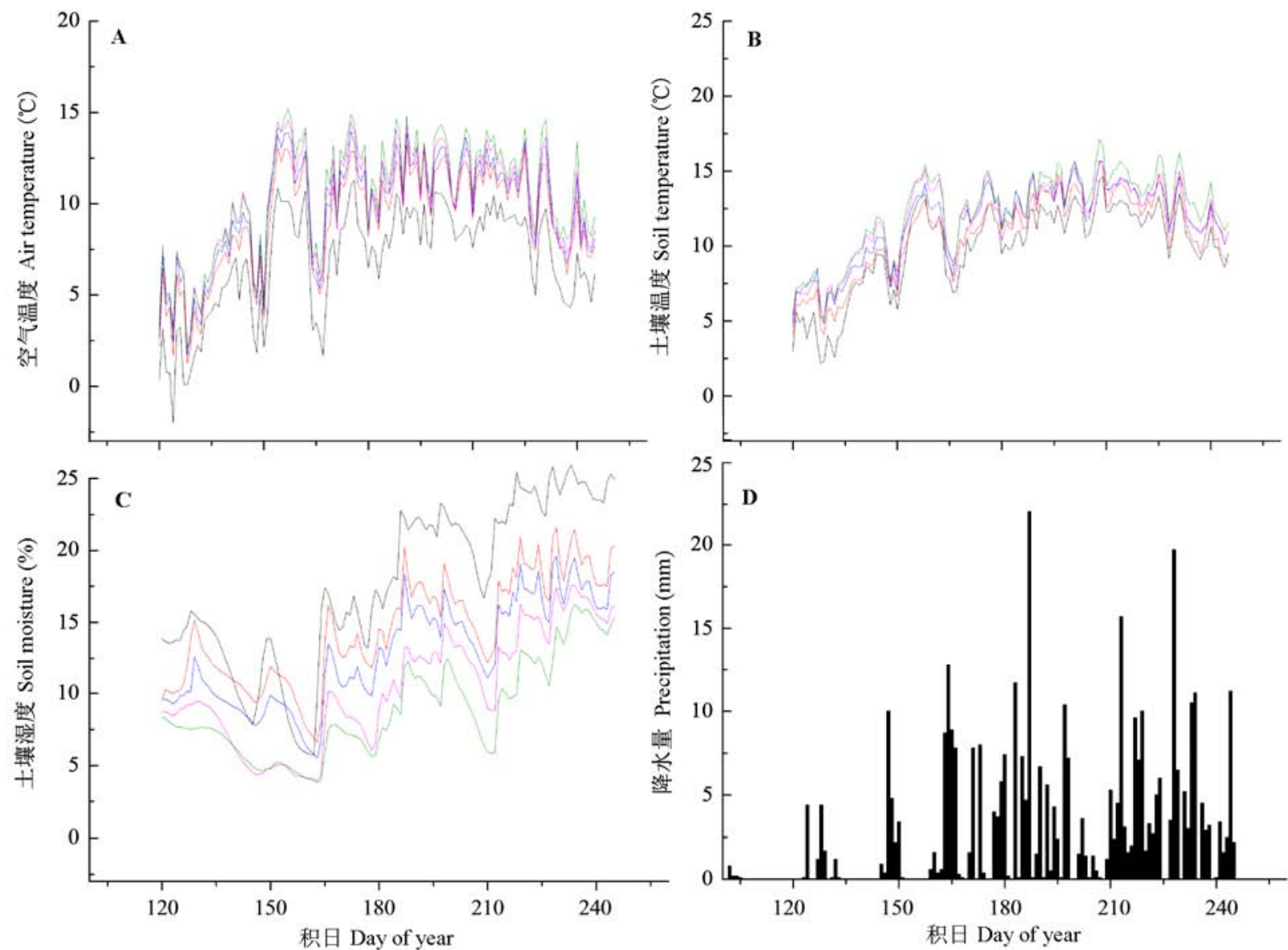

一对照 Control一增温T1 Warming T1 一增温 T2 Warming T2 增温 T3 Warming T3 增温 T4 Warming T4

图1 植物生长季不同增温处理下平均气温 $\left({ }^{\circ} \mathrm{C}, \mathbf{A}\right) 、 5 \mathrm{~cm}$ 土壤温度 $\left({ }^{\circ} \mathrm{C}, \mathbf{B}\right)$ 、土壤湿度 $(\%, \mathbf{C})$ 及降水量 $(\mathrm{mm}, \mathbf{D})$ 。

Fig. 1 Mean air temperature $\left({ }^{\circ} \mathrm{C}, \mathbf{A}\right)$, soil temperature at $5 \mathrm{~cm}$ depth $\left({ }^{\circ} \mathrm{C}, \mathbf{B}\right)$, soil moisture $(\%, \mathbf{C})$ at $5 \mathrm{~cm}$ depth and precipitation $(\mathrm{mm}, \mathbf{D})$ during the growing seasons under different warming treatments.

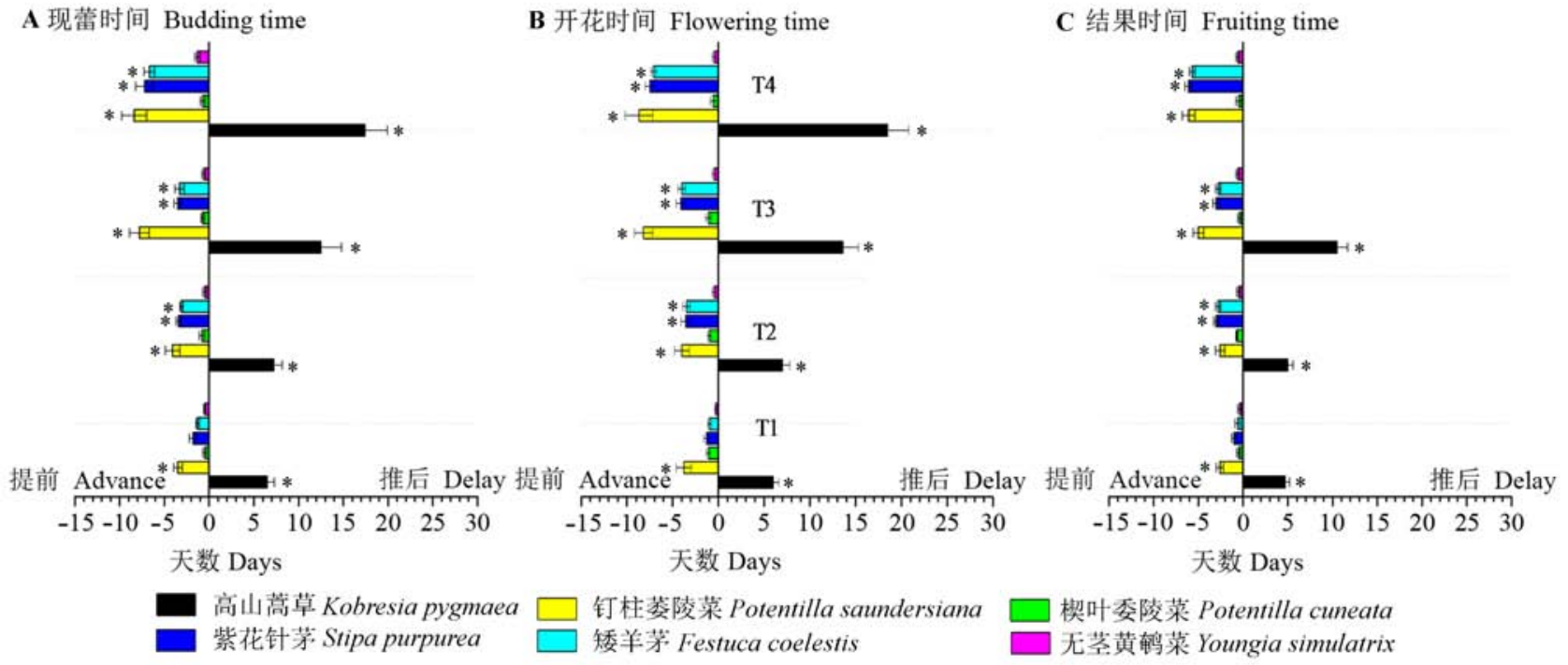

图2 增温处理对各物种的现蕾(A)、开花(B)和结果(C)时间的影响(平均值土标准误差)。图中正值代表与对照相比推后的天数, 负值代表与对照相比提前的天数; *代表增温处理与对照相比差异显著 $(p<0.05)$ 。

Fig. 2 Changes in the onset of budding, flowering and fruiting (in days) in four experimental treatments [i.e., T1, T2, T3, T4] compared with the control in 2014 (A, B, C) (mean \pm SE). A positive value indicates later budding, flowering or fruiting than the control; a negative value indicates earlier budding, flowering or fruiting than the control. Data are mean $\pm S E$ for advanced or delayed phenology, respectively. “*” indicates significant difference between treatment and the control. 
表2 增温处理和植物种类对现蕾、开花和结果时间及开花持续时间的影响

Table 2 Results ( $F$ value) of two-way ANOVA on the effects of warming, plant species and their interactions on budding time, flowering time, fruiting time and flowering duration

\begin{tabular}{|c|c|c|c|c|c|c|c|c|c|c|c|c|}
\hline \multirow{2}{*}{$\begin{array}{l}\text { 处理 } \\
\text { Treatment }\end{array}$} & \multicolumn{3}{|c|}{ 现蕾时间 Budding time } & \multicolumn{3}{|c|}{ 开花时间 Flowering time } & \multicolumn{3}{|c|}{ 结果时间 Fruiting time } & \multicolumn{3}{|c|}{ 开花持续 Flowering duration } \\
\hline & $\begin{array}{l}\text { 自由度 } \\
\text { Degree of } \\
\text { freedom }\end{array}$ & $F$ & $p$ & $\begin{array}{l}\text { 自由度 } \\
\text { Degree of } \\
\text { freedom }\end{array}$ & $F$ & $p$ & $\begin{array}{l}\text { 自由度 } \\
\text { Degree of } \\
\text { freedom }\end{array}$ & $F$ & $p$ & $\begin{array}{l}\text { 自由度 } \\
\text { Degree of } \\
\text { freedom }\end{array}$ & $F$ & $p$ \\
\hline 增温 Warming & 4 & 2.92 & 0.03 & & 1.84 & 0.13 & 3 & 1.13 & 0.35 & & 39.46 & 0.00 \\
\hline 物种 Species & 5 & 1153.39 & 0.00 & & 2567.89 & 0.00 & 5 & 2268.60 & 0.00 & & 589.42 & 0.00 \\
\hline $\begin{array}{l}\text { 增温×物种 } \\
\text { Warming } \times \text { species }\end{array}$ & 20 & 31.54 & 0.00 & & 24.97 & 0.00 & 15 & 12.74 & 0.00 & & 2.30 & 0.00 \\
\hline
\end{tabular}

表3 增温处理对各物种开花持续时间影响的 $F$ 检验

Table 3 Results of Fisher test showing the differences of the flowering duration for each species among treatments (T1, T2, T3, T4)

\begin{tabular}{llllc}
\hline 物种 Species & 增温T1 Warming T1 & 增温T2 Warming T2 & 增温T3 Warming T3 & 增温T4 Warming T4 \\
\hline 高山嵩草 Kobresia pygmaea & 0 & 0 & 1 & 1 \\
钉柱委陵菜 Potentilla saundersiana & 0 & 0 & 1 & 1 \\
楔叶委陵菜 Potentilla cuneata & 0 & 1 & 1 & 1 \\
紫花针茅 Stipa purpurea & 1 & 1 & 0 & 1 \\
矮羊茅 Festuca coelestis & 0 & 0 & 1 & 1 \\
无茎黄鹤菜 Youngia simulatrix & 0 & 1 & 1 \\
\hline
\end{tabular}

0 , 增温与对照的差异不显著 $(p>0.05) ; 1$, 增温与对照的差异显著 $(p<0.05)$ 。

" 0 " indicates that the means are not significantly different between warming treatments and the control $(p>0.05)$. " 1 " indicates that the means are significantly different between warming treatments and the control $(p<0.05)$.

高山嵩草的结果时间(图2), 在 $\mathrm{T} 1 、 \mathrm{~T} 2 、 \mathrm{~T} 3$ 处理下, 分别推迟了 $4.7 \pm 0.5 、 5.0 \pm 0.6 、 10.5 \pm 1.2 \mathrm{~d}(p<$ 0.05), T4处理下, 高山嵩草没有产生果实。与之相 反, 实验增温使钉柱委陵菜的结果时间显著提前 (图2), 在 $\mathrm{T} 1 、 \mathrm{~T} 2 、 \mathrm{~T} 3 、 \mathrm{~T} 4$ 处理下, 分别提前了 $2.6 \pm$ $0.4 、 2.7 \pm 0.3 、 5.0 \pm 0.6 、 6.1 \pm 0.7 \mathrm{~d}(p<0.05)$ 。 T2、 T3、T4处理使紫花针茅和矮羊茅的结果时间显著提 前, 分别提前了 $3.0 \pm 0.3 、 3.0 \pm 0.3 、 6.1 \pm 0.4 \mathrm{~d}$ 和 2.8 $\pm 0.3 、 2.8 \pm 0.6 、 5.7 \pm 0.5 \mathrm{~d}(p<0.05)$ 。实验增温对 楔叶委陵菜和无茎黄鹤菜的结果时间没有产生显著 影响(图2, $p>0.05$ )。

\section{5 实验增温对植物开花持续时间的影响}

双因素方差分析表明, 增温处理、植物种类及 其交互作用显著影响了植物的开花持续时间(表 $2, p$ $<0.05)$ 。与对照相比, 实验增温缩短了藏北高原植 物的开花持续时间(表5)。在 T3、T4处理下, 高山 嵩草和钉柱委陵菜的开花持续时间分别缩短了 1.6 $\pm 0.4 、 1.8 \pm 0.2 \mathrm{~d}$ 和 $1.4 \pm 0.5 、 2.5 \pm 0.6 \mathrm{~d}(p<0.05)$ 。 在T2、T3、T4处理下, 楔叶委陵菜和无茎黄鹤菜的 开花持续时间分别缩短了 $0.8 \pm 0.3 、 1.2 \pm 0.2 、 1.4 \pm$ $0.3 \mathrm{~d}$ 和 $0.5 \pm 0.2 、 0.7 \pm 0.3 、 0.8 \pm 0.3 \mathrm{~d}(p<0.05)$ 。在 $\mathrm{T} 1 、 \mathrm{~T} 2 、 \mathrm{~T} 3 、 \mathrm{~T} 4$ 处理下，紫花针茅的开花持续时间 分别缩短了 $1.3 \pm 0.2 、 1.9 \pm 0.3 、 2.0 \pm 0.3 、 2.3 \pm 0.5 \mathrm{~d}$ $(p<0.05)$ 。

\section{3 讨论}

在植物群落中, 不同物种物候期的差异是群落 水平上降低种间竞争并维持物种共存的重要机制 (Cleland et al., 2006), 而且植物开花和结实等物候 期的时间格局决定了植物群落的季相及其动态 $(\mathrm{Gu}$ et al., 1998)。植物物候被认为是气候变暖的“指纹”, 对温度升高的响应尤其敏感。我们的研究表明：增 温改变了藏北高寒草甸群落中多数物种的繁殖时 间，且不同种类对增温的响应不一致(提前、推后或 不变), 最终导致增温对开花和结果时间的影响不 显著, 而物种、物种和增温的交互作用显著改变了 植物的繁殖物候。在本研究区, 增温导致了土壤水 分胁迫, 尤其是在夏季季风来临之前(Dorji et al., 2013)。不同生活史性状的植物, 例如早开花和晚开 花植物, 及不同根系深度的植物对增温导致的表层 土壤水分胁迫的响应和敏感性不同。增温使浅根早花植物高山嵩草的现蕾时间推迟了 7-17天, 开花 时间推迟了 6-19天, 结果时间推迟了 5-11天。增温 导致的土壤水分蒸发和植物蒸腾作用提高, 严重制 约了该地区浅根植物的生长发育(Porporato et al., 2001; Klein et al., 2008)。高山嵩草又是早开花的物 
种，现蕾时间为5月29日(DOY: 152), 此时的夏季季 风刚开始(5月25日, DOY: 145), 降水相对较少, 增 温带来的土壤干旱胁迫更为严重, 因此, 早花植物 高山嵩草的现蕾和开花时间显著推迟，其物候对增 温的响应也更加敏感(Klein et al., 2008)。

对于相同的植物类型, 浅根-中花植物钉柱委 陵菜和楔叶委陵菜的物候对增温响应也不一致。增 温使钉柱委陵菜的现蕾时间提前了 4-8天, 开花时 间提前了 4-9天，结果时间提前了 3-6天; 而增温对 楔叶委陵菜的繁殖时间没有显著影响。对浅根-中花 植物而言, 其现蕾和开花时间在160-170天(DOY), 此时的夏季季风已经开始, 且降水相对较多, 可以 有效缓解增温带来的土壤水分减少, 因此, 中花植 物可能没有遭受严重的水分胁迫 (Dorji et al., 2013)。在此种情况下, 其他研究也报道增温可能提 前(Cleland et al., 2006)、推后(Hollister et al., 2005) 或不改变(Hoffmann et al., 2010)植物的物候。不同生 态系统中, 物种对于增温的响应表现出特异性, 人 们把物种之间出现的物候期响应差异归因于植物生 活型、传粉类型、开花时间等特性的差别(Fitter \& Fitter, 2002); 也有研究认为, 物种特异性是由于植 物光周期和春化作用对最低温的需求不同导致的 (Cook et al., 2012)。

对深根-晚花植物而言, 其现蕾和开花时间在 170-208天(DOY), 此时是生长季降水集中期, 且深 根性植物可以利用较深层的土壤水分, 因此, 与浅 根早花和中花植物相比, 深根-晚花植物的物候过 程较少受到土壤水分的限制。在高寒地区, 温度升 高可以缓减低温对植物生长发育带来的限制作用, 促进植物的生长(Dunne et al., 2003; 阿舍小虎, 2013)。因此, 在增温处理下, 紫花针茅和矮羊茅的 现蕾时间提前了 3-7天, 开花时间提前了 4-8天, 结 果时间提前了 3-6天。特别的是, 增温并没有显著改 变深根-晚花植物无茎黄鹤菜的繁殖时间。不同种类 植物物候对增温的响应快慢不同(Hoffmann et al., 2010), 一些种类的物候会快速响应增温, 而另一些 种类可能需要增温的累积效应(Arft et al., 1999)。例 如多数人认为早花植物可能比晚花植物对增温的响 应更敏感(Dunne et al., 2003; Wolkovich et al., 2012), 而在北美高草草原中夏季开花的植物对增 温的响应更敏感(Sherry et al., 2007), 在青藏高原早 春开花的植物对降温更敏感, 而中夏开花的植物对
增温更敏感(Wang et al., 2014)。因此, 本研究中无 茎黄鹤菜繁殖物候对增温的响应可能需要增温的累 积效应才能显现(Arft et al., 1999; Hoffmann et al., 2010)。

我们的研究还发现, 植物物候的各个阶段对增 温的敏感性不同。例如在增温处理下, 高山嵩草的 现蕾和开花时间平均推迟了大约 11 天, 而结果时间 平均推迟了大约 7 天; 钉柱委陵菜的现蕾和开花时 间平均提前了大约 6 天, 而结果时间平均提前了大 约 4 天。在藏北高原, 与果期相比, 现蕾和开花期对 增温的响应更加敏感(Hoffmann et al., 2010)。一些 研究还提出在增温的条件下, 即使花期发生了改变, 但由于植物生长发育的补偿机制，果期可能不变 (Post et al., 2008; Hoffmann et al., 2010), 植物的进 化过程对物候事件的影响不容忽视(Franks et al., 2007)。因此, 在预测和评估植物繁殖物候响应全球 增温的过程中, 还应当充分考虑植物生长发育的补 偿作用和进化过程(Post et al., 2008)。另外, 由于 OTCs自身的缺点, 如降低风速等, 可能会对一些风 媒传粉的物种有影响, 例如高山嵩草, 因此OTCs本 身可能会影响一些物种的结果时间。

在全球变暖的背景下, 植物的生长季延长(Piao et al., 2006)或缩短(Chmielewski \& Rötzer, 2001)均 有报道。在同一草地类型, 一些物种的繁殖期延长, 而另外一些物种的繁殖期缩短(Sherry et al., 2007)。 在藏北高原, 增温使植物的开花持续时间缩短。例 如高山嵩草开花持续期平均缩短了 1.7 天, 紫花针茅 开花持续期缩短了 1.9 天, 无茎黄鹤菜的开花持续期 缩短了 0.7 天。在藏北高原增温导致土壤水分胁迫, 在干旱缺水的条件下, 植物加快繁殖时间可能是应 对逆境的一种适应策略。在本研究中, 增温处理导 致了不同类型植物的繁殖时间发生明显偏移, 这可 能会影响群落中相邻开花种类的繁殖重叠时间, 进 而可能改变它们之间的竞争关系(Goldman \& Willson, 1986; Kliber \& Eckert, 2004), 对植物的繁殖产 生重大影响。本研究也预示着, 在未来更热更干的 生长季, 青藏高原高寒草甸系统的植物物候格局可 能会被重塑。

基金项目 国家重大科学研究计划(2013CB956302 和2010CB950603)、国家自然科学基金(41571195) 和中国科学院“西部之光”项目。 
致谢 特别感谢中国科学院地理科学与资源研究所 博士研究生黄珂、刘瑶杰、俎佳星等在西藏那曲野 外观测工作中给予的大力帮助。

\section{参考文献}

Ashe XH (2013). Effects of Warming and Precipitation Regime on Plant Phenology and Productivity in an Alpine Meadow, Northwestern Sichuan, China. Master degree dissertation, Chengdu University of Technology, Chengdu. (in Chinese with English abstract) [阿舍小虎 (2013). 模拟 增温与降水改变对川西北高寒草甸植物物候及初级生 产力的影响. 硕士学位论文, 成都理工大学, 成都.]

Arft AM, Walker MD, Gurevitch J, Alatalo JM, Bret-Harte MS, Dale M (1999). Response patterns of tundra plant species to experimental warming: A meta-analysis of the international tundra experiment. Ecological Monographs, 69, 491-511.

Bjorkman AD, Elmendorf SC, Beamish AL, Vellend M, Henry GHR (2015). Contrasting effects of warming and increased snowfall on Arctic tundra plant phenology over the past two decades. Global Change Biology, 21, 4651-4661.

Chmielewski FM, Rötzer T (2001). Response of tree phenology to climate change across Europe. Agricultural and Forest Meteorology, 108, 101-112.

Cleland EE, Chiariello NR, Loarie SR, Mooney HA, Field CB (2006). Diverse responses of phenology to global changes in a grassland ecosystem. Proceedings of the National Academy of Sciences of the United States of America, 103, 13740.

Cook BI, Wolkovich EM, Parmesan C (2012). Divergent responses to spring and winter warming drive community level flowering trends. Proceedings of the National Academy of Sciences of the United States of America, 109, 9000-9005.

Dorji T, Totland Ø, Moe S, Hopping KA, Pan JB, Klein JA (2013). Plant functional traits mediate reproductive phenology and success in response to experimental warming and snow addition in Tibet. Global Change Biology, 19, 459-472.

Dunne JA, Harte J, Taylor KJ (2003). Subalpine meadow flowering phenology responses to climate change: Integrating experimental and gradient methods. Ecological Monographs, 73, 69-86.

Fitter AH, Fitter RSR (2002). Rapid changes in flowering time in British plants. Science, 296, 1689-1691.

Franks SJ, Sim S, Weis AE (2007). Rapid evolution of flowering time by an annual plant in response to a climate fluctuation. Proceedings of the National Academy of Sciences of the United States of America, 104, 1278-1282.

Goldman DA, Willson MF (1986). Sex allocation in functionally hermaphroditic plants: A review and critique. Botanical Review, 52, 157-194.
Gu S, Hui D, Bian A (1998). The contraction-expansion algorithm and its use in fitting nonlinear equations. International Journal of Biomathematics, 13, 426-434.

Hoffmann AA, Camac JS, Williams RJ, Papst W, Jarrad FC, Wahren C-H (2010). Phenological changes in six Australian subalpine plants in response to experimental warming and year-to-year variation. Journal of Ecology, 98, 927-937.

Hollister RD, Webber PJ, Bay C (2005). Plant response to temperature in northern Alaska: Implications for predicting vegetation change. Ecology, 86, 1562-1570.

IPCC (Intergovernmental Panel on Climate Change) (2007). Contribution of working group I to the fourth assessment report of the intergovernmental panel on climate change. In: Solomon S, Qin DH, Manning M, Marquis M, Chen ZL, Averyt K, Tignor M, Miller HL eds. Climate Change 2007: The Physical Science Basis. Cambridge University Press, Cambridge, UK.

Klein JA, Harte J, Zhao XQ (2008). Decline in medicinal and forage species with warming is mediated by plant traits on the Tibetan Plateau. Ecosystems, 11, 775-789.

Kliber A, Eckert CG (2004). Sequential decline in allocation among flowers within Inflorescences: Proximate mechanism and adaptive significance. Ecology, 85, 1675-1687.

Li YH, Han GD, Wang Z, Zhao ML, Wang ZW, Zhao HB (2014). Influences of warming and nitrogen addition on plant reproductive phenology in Inner Mongolia desert steppe. Chinese Journal of Ecology, 33, 849-856. (in Chinese with English abstract) [李元恒, 韩国栋, 王珍, 赵萌 莉, 王正文, 赵鸿涁 (2014). 增温和氮素添加对内蒙古 荒漠草原植物生殖物候的影响. 生态学杂志, 33, 849-856.]

Piao SL, Fang JY, Zhou LM, Philippe C, Zhu B (2006). Variations in satellite-derived phenology in China's temperate vegetation. Global Change Biology, 12, 672-685.

Porporato A, Laio F, Ridolfi L, Rodriguez-Iturbe I (2001). Plants in water-controlled ecosystems: Active role in hydrologic processes and response to water stress: III. Vegetation water stress. Advances in Water Resources, 24, 725-744.

Post ES, Pedersen C, Wilmers CC, Forchhammer MC (2008). Phenological sequences reveal aggregate life history response to climatic warming. Ecology, 89, 363-370.

Price MV, Waser NM (1998). Effects of experimental warming on plant reproductive phenology in a subalpine meadow. Ecology, 79, 1261-1271.

Richards FJ (1959). A flexible growth function for empirical use. Journal of Experimental Botany, 10, 290-301.

Rutishauser T, Stockli R, Harte J, Kueppers L (2012). Climate change: Flowering in the greenhouse. Nature, 485, 448-449.

Sherry RA, Zhou XH, Gu SL, Arnone JA, Schimel DS, 
Verburg PS, Wallace LL, Luo YQ (2007). Divergence of reproductive phenology under climate warming. Proceedings of the National Academy of Sciences of the United States of America, 104, 198-202.

Springate DA, Kover PX (2014). Plant responses to elevated temperatures: A field study on phenological sensitivity and fitness responses to simulated climate warming. Global Change Biology, 20, 456-465.

Visser ME, Both C (2005). Shifts in phenology due to global climate change: The need for a yardstick. Proceedings of the Royal Society Biological Sciences, 272, 2561-2569.

Wang SP, Meng FD, Duan JC, Wang YF, Cui XY, Piao SL, Niu HS, Xu GP, Luo CY, Zhang ZH, Zhu XX, Shen MG, Li YN, Du MY, Tang YH, Zhao XQ, Ciais PB, Kimball B, Peñuelas J, Janssens IA, Cui SJ, Zhao L, Zhang FW (2014). Asymmetric sensitivity of first flowering date to warming and cooling in alpine plants. Ecology, 95,
3387-3398.

Wolkovich EM, Cook BI, Allen JM, Crimmins TM, Betancourt JL, Travers SE, Pau S, Regetz J, Davies TJ, Kraft NJB, Ault TR, Bolmgren K, Mazer SJ, McCabe GJ, McGill BJ, Parmesan C, Salamin N, Schwartz MD, Cleland EE (2012). Warming experiments underpredict plant phenological responses to climate change. Nature, 485, 494-497.

Xia J, Wan S (2013). Independent effects of warming and nitrogen addition on plant phenology in the Inner Mongolian steppe. Annals of Botany, 111, 1207-1217.

Yu H, Luedeling E, Xu J (2010). Winter and spring warming result in delayed spring phenology on the Tibetan Plateau. Proceedings of the National Academy of Sciences of the United States of America, 107, 22151-22156.

责任编委: 倪 健 责任编辑: 王 葴

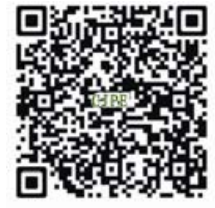

植物生态学报官网

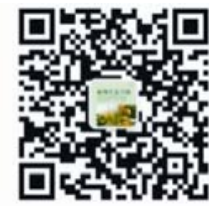

微信订阅号

期刊及学科

相关信息发布

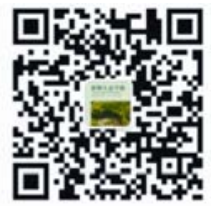

微信服务号

稿件状态查询

全文检索汶览 